\title{
Efficient base editing with high precision in rabbits using YFE-BE4max
}

\author{
Zhiquan Liu', Siyu Chen ${ }^{1}$, Huanhuan Shan ${ }^{1}$, Yingqi Jia ${ }^{1}$, Mao Chen ${ }^{1}$, Yuning Song ${ }^{1}$, Liangxue Lai ${ }^{1,2,3,4}$ and Zhanjun Li $^{1}$
}

\begin{abstract}
Cytidine base editors, composed of a cytidine deaminase fused to Cas9 nickase, enable efficient C-to-T conversion in various organisms. However, current base editors suffer from severe trade-off between editing efficiency and precision. Here, based on rationally mutated cytidine deaminase domain, we develop a new base editor, YFE-BE4max, effectively narrow the editing width to as little as approximately three nucleotides while maintaining high efficiency in rabbits. Moreover, YFE-BE4max successfully mediated the Tyr p. Q68Stop and Lmna p. G607G mutation in F0 rabbit with high efficiency and precision, which precisely recapitulates the pathological features of human OCA1 and HGPS, respectively. Collectively, YFE-BE4max system provide promising tools to perform efficient base editing with high precision in rabbits and enhances its capacity to precisely model human diseases.
\end{abstract}

\section{Introduction}

The clustered regularly interspaced short palindromic repeat (CRISPR) system has exhibited powerful genome manipulation capability in various organisms ${ }^{1,2}$. Base editing is a revolutionary technology based on the CRISPR platform, which can achieve targeted C-to-T conversion without generating DNA double-strand breaks or requiring a donor template ${ }^{3}$. The most common base editing system, base editor 3 (BE3), consists of rat APOBEC1 (rA1) fused with a Streptococcus pyogenes Cas9 (SpCas9) nickase and uracil glycosylase inhibitor ${ }^{3}$. Efficient editing by $\mathrm{BE} 3$ requires the presence of a protospacer-adjacent motif (PAM) of NGG that places the target $\mathrm{C}$ within an $\sim 5$-nucleotide window near the PAM-

\footnotetext{
Correspondence: Liangxue Lai (lai_liangxue@gibh.ac.cn) or

Zhanjun Li (lizj_1998@jlu.edu.cn)

${ }^{1}$ Key Laboratory of Zoonosis Research, Ministry of Education, College of Animal Science, Jilin University, Changchun 130062, China

${ }^{2}$ CAS Key Laboratory of Regenerative Biology, Guangdong Provincial Key Laboratory of Stem Cell and Regenerative Medicine, South China Institute for Stem Cell Biology and Regenerative Medicine, Guangzhou Institutes of

Biomedicine and Health, Chinese Academy of Sciences, Guangzhou 510530, China

Full list of author information is available at the end of the article.

These authors contributed equally: Zhiquan Liu, Siyu Chen, Huanhuan Shan

Edited by $Y$. Shi
}

distal end of the protospacer (positions 4-8, counting the PAM as positions 21-23) in human cells ${ }^{3,4}$.

BE3 can potentially induce unwanted C-to-T substitutions when more than one $\mathrm{C}$ is present in the large $\sim 5$ nucleotide window, which can negatively affect the precision of targeted base editing. Therefore, such system is not ideal for precise disease modeling and gene therapy when accurate single $C$ substitution is required. To overcome this limitation, Liu et al optimized rA1 with mutant deaminase domains (YE base editors), representatively termed YE1 (W90Y + R126E) and YEE (W90Y + R126E + R132E), to effectively narrow the width of the editing window from $\sim 5$ nucleotides to as little as 1-2 nucleotides in human cells ${ }^{4}$. YEE-BE3 showed better accuracy than YE1-BE3, but has lowered editing efficiency at target loci ${ }^{4,5}$.

In this report, by rationally engineering Cas9-cytidine deaminase fusions, we optimized cytidine base editors with high precision. In addition, these new base editors were successfully applied to produce Founder (F0) rabbits, demonstrating their high efficiency and precision in inducing $\mathrm{C}$-to- $\mathrm{T}$ conversions at multiple endogenous loci in organisms.

\section{(c) The Author(s) 2020}

(c) (i) Open Access This article is licensed under a Creative Commons Attribution 4.0 International License, which permits use, sharing, adaptation, distribution and reproduction cc) in any medium or format, as long as you give appropriate credit to the original author(s) and the source, provide a link to the Creative Commons license, and indicate if changes were made. The images or other third party material in this article are included in the article's Creative Commons license, unless indicated otherwise in a credit line to the material. If material is not included in the article's Creative Commons license and your intended use is not permitted by statutory regulation or exceeds the permitted use, you will need to obtain permission directly from the copyright holder. To view a copy of this license, visit http://creativecommons.org/licenses/by/4.0/. 


\section{Results}

Comparison of C-to-T base editing using BE4-Gam and other mutated deaminase-Cas9 fusions in rabbit embryos

Our previous study demonstrated that BE3 system can be used to mimic human pathologies by efficiently mediate $\mathrm{C}$-to- $\mathrm{T}$ conversions in rabbits ${ }^{6}$. However, it frequently induce high proportions of bystander mutations at target loci, especially at Tyr-1 p. Q68Stop and Lmna-1 p. $\mathrm{G}_{607 \mathrm{G}^{6}}$. The results of deep sequencing analysis indicated that unwanted $\mathrm{C}$-to- $\mathrm{T}$ mutations were observed in a large width from $\mathrm{C} 2$ to $\mathrm{C} 11$ or from $\mathrm{C} 6$ to $\mathrm{C} 17$ at Tyr-1 or Lmna-1, respectively (Fig. S1). In particular, a high proportion of bystander C-to- $\mathrm{T}$ mutations lead to changes in amino acids such as bystander C3 $(50.83 \%$, p. P67L) at Tyr-1 and bystander C11 (77.00\%, p. S609F) at Lmna-1, thus may potentially confound the correspondence between the genotype and phenotype of mutants (Figs. 1a and S1). The bystander mutations may arise from the relatively wide editing window of $\mathrm{BE} 3$, and the high activity of APOBEC1 likely contributes to the deamination of multiple Cs per DNA-binding event, consistent with previous reports in human cells ${ }^{3}$.

To reduce the bystander mutations, we first tested the current representative YE1 and YEE systems in rabbit embryos at Tyr-1 and Lmna-1 as a proof of concept. The YE1-BE4-Gam and YEE-BE4-Gam vectors were constructed by introducing the corresponding point mutations into BE4-Gam, a new version of base editor that has been demonstrated to have identical efficiency to traditional BE3 and higher product purity ${ }^{6,7}$ (Fig. 1b). Base editing frequencies were evaluated from Sanger sequence chromatograms of each blastocyst using EditR, a robust and inexpensive base editing quantification software has been widely used in previous reports ${ }^{6,8,9}$. Notably, YE1BE4-Gam maintained activity comparable to that of BE4Gam at the target C5 $(80.47 \pm 13.25 \%$ versus $75.28 \pm$ 9.38\%), whereas minimized deamination activity at other positions, thus narrowing the editing width from $\mathrm{C} 3$ to $\mathrm{C} 5$ at Tyr-1 (Fig. 1c). In addition, at Lmna-1, YE1-BE4-Gam almost eliminated the bystander activity at $\mathrm{C} 11$ and $\mathrm{C} 12$ but did modestly reduce the editing frequency at target C6 $(52.95 \pm 7.14 \%$ versus $75.55 \pm 10.86 \% ; p=0.11)$ (Fig. $1 \mathrm{~d})$. However, YEE-BE4-Gam showed significantly reduced editing efficiency at both target loci $(22.52 \pm 8.21 \%$; $p<$ 0.01 at Tyr-1 and $11.10 \pm 5.07 \% ; p<0.001$ at Lmna-1) although nearly free from bystander mutations (Fig. 1c, d). This outcome is consistent with the low efficiency of YEE variant that previously reported in cultured cells ${ }^{4,5,10}$.

A recently developed base editor, BE4max, can significantly increase editing efficiency by modifying nuclear localization signals and codon usage ${ }^{11}$. Subsequently, we exploited this method to optimize the efficiency of $\mathrm{YE}$ base editors, and constructed YE1-BE4max and YEEBE4max systems (Fig. 1b). In comparison with YE1-BE4-
Gam, YE1-BE4max showed higher activity at Tyr-1 $(86.05 \pm 5.46 \%$ versus $80.47 \pm 13.25 \%)$ and Lmna-1 $(64.07 \pm 5.02 \%$ versus $52.95 \pm 7.14 \%) \quad$ (Fig. 1c, d). The YEE-BE4max showed surpassing activity at both loci compared with that of YEE-BE4-Gam, but its editing efficiency is relatively inferior $(43.88 \pm 13.44 \%$ at $T y r-1$ and $25.46 \pm 5.52 \%$ at Lmna-1) compared with that of conventional BE4-Gam (Fig. 1c, d). In addition, all five base editors exhibited relatively low frequencies of indels and non-C-to- $\mathrm{T}$ conversions, consistent with the high product purity of the BE4 system in previous reports 6,7 (Fig. S2). Overall, these results indicated that YE1 base editors (YE1-BE4-Gam and YE1-BE4max) narrowed the editing window to $\sim \mathrm{C} 3-\mathrm{C} 6$ with comparable efficiency, and YEE base editors (YEE-BE4-Gam and YEE-BE4max) narrowed the editing window to $\sim \mathrm{C} 5-\mathrm{C} 6$ with notably decreased activity at the two loci (Fig. 1e, f).

\section{Optimization of deaminase domain improves efficiency and precision}

The point mutations in rA1 of YE1 and YEE were designed to slow its kinetic rate, thereby reduced the catalytic efficiency of the deaminase domain ${ }^{4}$. In fact, W90Y and R126E mutations in YE1 can specifically narrow the size of editing window while maintain efficiency, but an additional R132E mutation in YEE can dramatically reduce the editing efficiency. Thus, rationally engineering cytidine deaminases by replacing the deleterious R132E mutation may be an effective strategy to improve the base editors' efficiency and precision simultaneously. Moreover, the mutation of residue Y130 partially reduced the deamination activity of human APOBEC3A (hA3A), thereby narrowing the editing width in a recent study ${ }^{12}$. $\mathrm{Y} 130$ in hA3A, equivalent to $\mathrm{Y} 120$ in $\mathrm{rA} 1$, is near the active center and crucial for contacting the target cytosine $^{13,14}$. Furthermore, Y120 in rA1 is a highly conserved site among multiple cytidine deaminases, as shown by alignment of the amino-acid sequences (Fig. 2a).

Based on these findings, we introduced similar Y120F mutations into rA1 (Y120F-BE4max) (Fig. 2b). Four target sites with a subset of Cs present in the activity window were selected to verify the hypothesis. The control group, BE4-Gam, showed a large editing window mainly from C4 to $C 9(\sim 6 \mathrm{nt})$ and even induced the widest range of mutations spanning from $\mathrm{C} 4$ to $\mathrm{C} 15$ at Tyr-2 (Fig. 2c-f). In contrast, YE1-BE4max exhibited ideal efficiency comparable to that of BE4-Gam, but narrowed the editing width to $\sim 4 \mathrm{nt}(\mathrm{C} 4-\mathrm{C} 7)$ at $T y r-2$ and $\sim 3 \mathrm{nt}$ at the other three sites (Fig. 2c-f). YEE-BE4max narrowed the editing window to $\sim 3$-nt (C4-C6) at Tyr-2 and Tyr-3, even to $\sim 1$ nt (C6) at Mstn-1 and Mstn-2, but its editing efficiency is significantly reduced. Notably, modified Y120F-BE4max maintained high activity and decreased bystander mutations with narrowed editing window $(\sim 3-4 \mathrm{nt})$ at target 


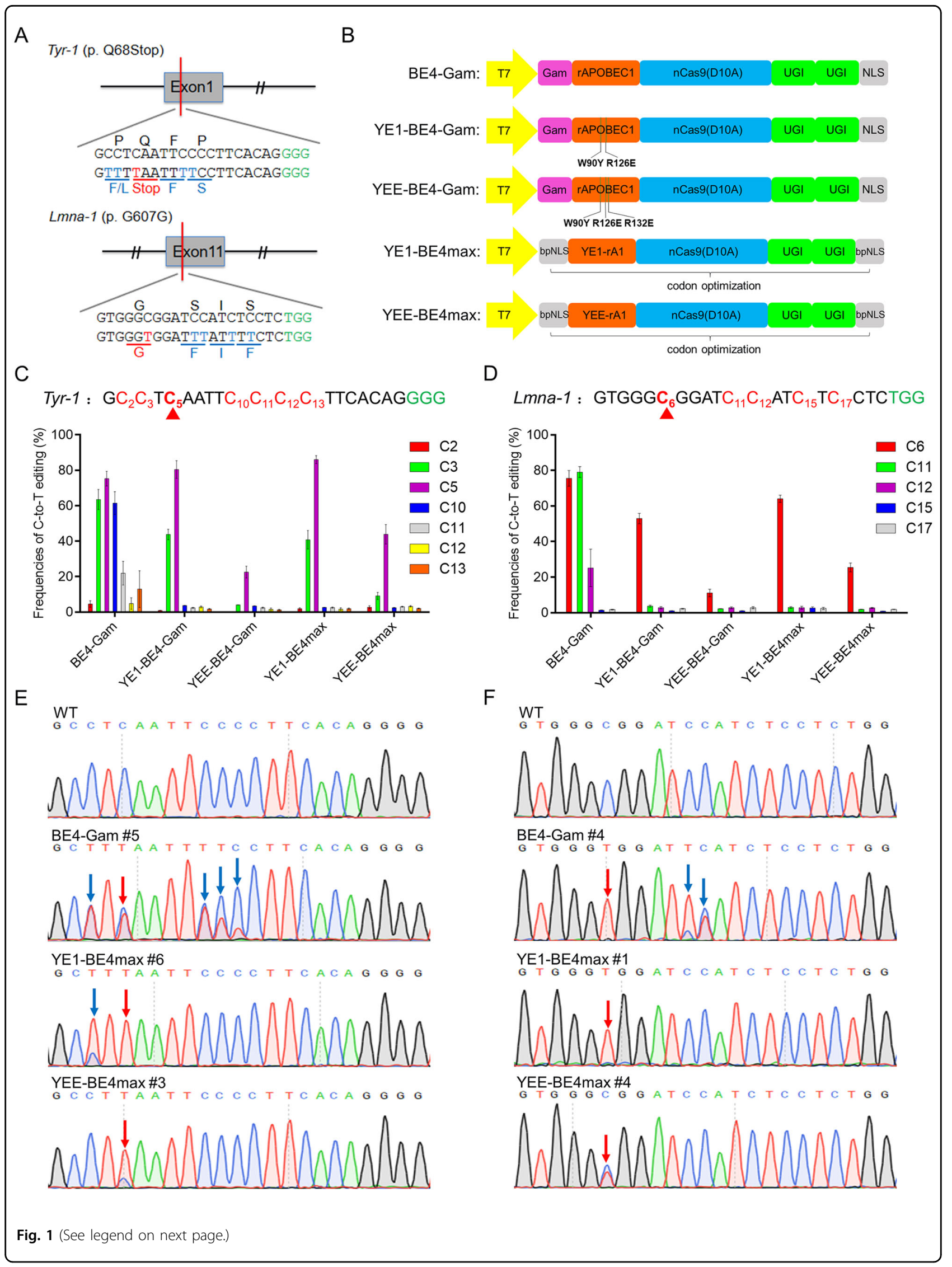


(see figure on previous page)

Fig. 1 Comparison of C-to-T base editing using BE4-Gam and other mutated deaminase-Cas 9 fusions in rabbit embryos. a The target sequence at the Tyr- 1 and $L m n a-1$ sites. Target sequence (black), PAM region (green). The target C-to-T-editing sites or potential bystander mutations are marked in red or blue, respectively. The relevant codon identities at the target site are presented under the DNA sequence. b Schematic representation of the five base editors' architecture. The point mutations that mutated in $\mathrm{AAPOBEC1}$ are indicated by the green lines. YE1 (W90Y + R126E), YEE (W90Y+R126E+R132E). c, d Frequencies of targeted single C-to-T conversion at Tyr-1 c and Lmna-1 d by five base editors in rabbit embryos. The red triangle indicates the target $\mathrm{C}$ of the desired mutation. Target sequence (black), PAM region (green), mutated Cs (red, counting the PAM as positions 21-23). e, f Representative sequencing chromatograms at Tyr- 1 e and $L m n a-1 \mathbf{f}$ of edited rabbit blastocyst using three base editors. Targeted base editing (red arrows), bystander base editing (blue arrows). WT, wild type.

sites (Fig. 2c-f), which indicates that Y120F mutation has a similar effect of narrowing editing window as the combined effect of W90Y and R126E in YE1, and does not affect editing efficiency. Encouraged by this result, we further combined the Y120F and YE1 mutations to produce a triply mutated base editor, termed YFE-BE4max (W90Y + Y120F + R126E) (Fig. 2b). Remarkably, YFEBE4max showed similar maximal editing efficiency as Y120F-BE4max, but substantially narrowed editing window width to $\sim 3 \mathrm{nt}$ on both Tyr-2 and Tyr-3 (Fig. 2c, d). In particular, induced C-to- $\mathrm{T}$ conversion at $\mathrm{C} 6$ almost accurately, with few bystander mutations at both Mstn-1 and Mstn-2 (Fig. 2e, f). Moreover, YFE-BE4max efficiently induced precise base editing at target Cs at both Tyr-1 and Lmna-1 with minimized bystander activities in surrounding regions (Fig. $2 \mathrm{~g}-\mathrm{i}$ ). Furthermore, by conducting detailed analysis, the YFE-BE4max can efficiently induce base editing in a narrower window ( $3 \mathrm{nt}$, positions $4-6$ in the sgRNA target site) compared with that of initial BE4-Gam ( 9 nt, positions 3-11) (Fig. S3). Taken together, these results demonstrated that YFE-BE4max can induce efficient base editing within a narrow window $(\sim 3 \mathrm{nt})$ in rabbit embryos, thus it can serve as a powerful tool to mediate targeted point mutations.

\section{Precise base conversion at Tyr to recapitulate human albinism}

To further demonstrate the use of YFE-BE4max to generate F0 mutant rabbits, we produced albino and premature aging rabbits by mutate Tyr and Lmna gene, respectively. The TYR gene is the causal gene of human oculocutaneous albinism type 1 (OCA1) ${ }^{15}$, a single C.G to $\mathrm{T} \cdot \mathrm{A}$ base pair conversion was designed in exon 1 of the rabbit Tyr gene for the purpose of yielding a premature stop codon (p. Q68stop) (Fig. 3a). After microinjection of YFE-BE4max-encoding mRNA and single guide RNA (sgRNA), rabbit embryos were transplanted into surrogate mother. Five pups were finally obtained, and the result of targeted deep sequencing showed that all these newborn rabbits (100\%) were homozygous with nonsense mutation at the target site (Fig. 3b and Table 1). Notably, the T3 mutant harbored only the mutant allele at target C5 to induce the completely precise p. Q68stop mutation of the rabbit Tyr gene (Fig. 3b-d), which is arduous for BE3 to achieve due to rAl's excessive bystander activity ${ }^{6}$. Though slight bystander mutations $(4.8 \%$ at $\mathrm{C} 2,13.6 \%$ at $\mathrm{C} 3$, and $1.2 \%$ at $\mathrm{C} 10$ ) proximal to the targeted $\mathrm{C} 5$ were also observed, the frequency of unwanted mutation was significantly reduced by YFE-BE4max in compare with $\mathrm{BE}^{6}{ }^{6}$ (Fig. 3e). Moreover, few C-to-A substitutions were also observed in one mutant rabbit $(1 \%, \mathrm{~T} 1)$, whereas no indels were detected, further demonstrating the high product purity of YFE-BE4max (Fig. 3b). No obvious offtarget mutations were detected at potential off-target sites in mutant rabbits by using Sanger sequencing (Fig. S6a). All five pups (100\%) showed a systemic albino phenotype (Fig. 3f). In addition, histological haematoxylin and eosin (H\&E) staining revealed the absence of melanin in the skin and lens of mutants, but not in the wild type (WT) rabbit (Figs. 3g and S4). These results showed efficient base substitution at Tyr-1 by the YFE-BE4max and its potential to decrease unwanted bystander mutations in rabbits.

\section{Base conversion at Lmna to precisely mimic the human HGPS mutation}

A de novo point mutation (p. G608G) in the LMNA gene can lead human classical Hutchinson-Gilford progeria syndrome (HGPS), which is a rare genetic disorder that characterized by premature and rapid aging shortly after birth ${ }^{16,17}$. Here, a single C-to- $\mathrm{T}$ conversion in exon 11 of Lmna gene was designed to induce p.G607G mutation in rabbit, which is equivalent to the p.G608G mutation in human HGPS $^{6}$ (Fig. 4a). Notably, targeted point mutation at the target $\mathrm{C} 6$ was successfully induced all of six (100\%) rabbits with editing frequencies from 57 to $98 \%$ (Fig. $4 \mathrm{~b}$ and Table 1). Moreover, a few bystander mutations were only detected in the L6 mutant ( $7 \%$ at C11) (Fig. 4b). In particular, the L5 mutant harbored only the desired C-to-T conversion at target C6 with 98\% mutant frequency, enabling it to induce highly efficient and accurate p. G607G mutation (Fig. 4b-d). The analysis of deep sequencing results showed that YFE-BE4max significantly reduced bystander mutations at the Lmna locus in compare with previous BE3 system (Fig. 4e). Similarly, no indels, non-C-to-T conversions or off-target mutations were detectable in all mutant rabbits (Figs. 4b and S6b). 


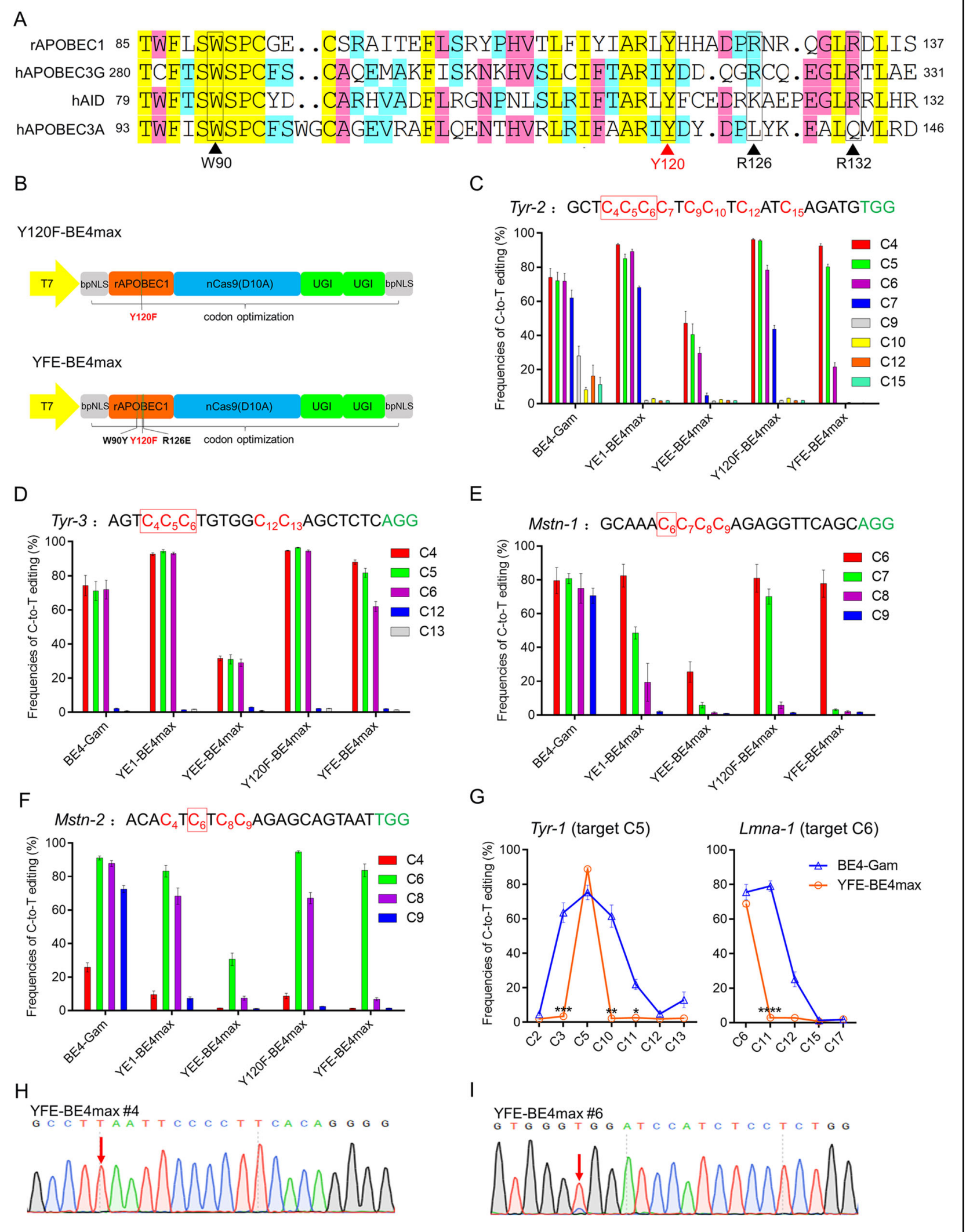

Fig. 2 (See legend on next page.) 
(see figure on previous page)

Fig. 2 Base editors with rationally engineered cytidine deaminase domains exhibit narrowed editing windows with maintained efficiency in rabbit embryos. a Amino-acid alignments of $\mathrm{APPOBEC} 1$ with three human APOBEC/AID family members. W90, R126, and R132 or Y120 are shown in black or red, respectively. $\mathbf{b}$ Schematic representation of Y120F-BE4max and YFE-BE4max. c-f Frequencies of single C-to-T conversion at Tyr-2 c, Tyr$3 \mathbf{d}$, Mstn-1 e, and Mstn-2 $\mathbf{f}$ by five base editors in rabbit embryos. Target sequence (black), PAM region (green), mutated Cs (red, counting PAM as positions 21-23). The red rectangle indicates the core window of YFE-BE4max. $\mathbf{g}$ Comparison of frequencies of single C-to-T conversions using BE4Gam and YFE-BE4max at Tyr-1 and $L m n a-1$ in rabbit embryos. $\mathbf{h}$, i Representative sequencing chromatograms at Tyr- $1 \mathbf{h}$ and $L m n a-1 \mathbf{i}$ of edited rabbit blastocyst using YFE-BE4max. Targeted base editing (red arrows).

Table 1 Generation of targeted mutations in F0 rabbits.

\begin{tabular}{|c|c|c|c|c|c|c|c|}
\hline \multirow[t]{2}{*}{ System } & \multirow[t]{2}{*}{ Target site } & \multirow{2}{*}{$\begin{array}{l}\text { Embryos } \\
\text { transferred }\end{array}$} & \multirow{2}{*}{$\begin{array}{l}\text { No. of } \\
\text { offspring }\end{array}$} & \multicolumn{4}{|c|}{ Mutant ratio (\%) } \\
\hline & & & & $\begin{array}{l}\text { No. of } \\
\text { mutants }\end{array}$ & $\begin{array}{l}\text { Frequencies of precise } \\
\text { target editing }\end{array}$ & No. of indels & $\begin{array}{l}\text { No. of non-C-to-T } \\
\text { editing }\end{array}$ \\
\hline \multirow[t]{2}{*}{ YFE-BE4max } & Tyr-1 & 34 & 5 & $5(100)$ & (57-98) & $0(0)$ & $1(20)$ \\
\hline & Lmna-1 & 42 & 6 & $6(100)$ & $(57-98)$ & $0(0)$ & $0(0)$ \\
\hline
\end{tabular}

Mutations in the $L M N A$ gene are associated with a wide range of human genetic disorders, and different mutations may cause discrepant phenotypes ${ }^{18}$. BE3 system is likely to induce high frequency of bystander mutation at C11 thus mediating p. S609F missense mutation, which may potentially affect the phenotype of mutants. Actually, the all F0 mutant rabbits produced by BE3 died within 12 weeks due to a lethal phenotype, but three mutant rabbits generated by YFE-BE4max were still alive at 16 weeks to date (Fig. 4f). This result indicated that the inaccurate p. G607G mutation with high frequencies of bystander mutations using BE3 could not faithfully reflect the phenotype of human HGPS. The p.G607G mutation results in the deletion of 150 nucleotides (50 aa) at the $C$ terminal of exon 11 by creating a cryptic splice donor site, thus producing a mutant lamin A protein, termed "progerin"16. As expect, the RT-PCR analysis and subsequent Sanger sequencing showed the presence of truncated products with 150 nucleotides deletion in mutants, which do not exist in WT rabbits (Fig. 4g, h, and S5). Furthermore, the typical phenotype of marked reductions in growth rate and survival rate (Fig. 4f), short stature (Fig. 4i, j), thin skin, and loss of subcutaneous fat (Fig. 4k) were also observed in the mutants. These observations are completely consistent with RNA mis-splicing and corresponding phenotypes observed in human HGPS patients ${ }^{16,17}$. Over all, with great editing efficiency and minimized bystander activity, YFE-BE4max successfully mediated Lmna-1 mutation in F0 rabbit, which precisely recapitulate pathological features of classical human HGPS.

\section{Discussion}

In this study, we demonstrated that the YE base editors make a compromise between efficiency and precision, and especially YEE base editors significantly reduce editing efficiency. To overcome this limitation, we developed an optimized rA1-nCas9 fusion, YFE-BE4max, effectively narrow the editing width to as little as $\sim 3 \mathrm{nu}$ while maintaining high efficiency in rabbits by rationally engineering the deaminase domain. In addition, the Y120FBE4max can efficiently induce C-to- $\mathrm{T}$ conversions with narrowed $\sim 3-4 \mathrm{nu}$ windows, similar to YE1-BE4max. Moreover, YFE-BE4max was used to induce site-specific single-base substitutions with $100 \%$ efficiency and reduced bystander mutations in generating $\mathrm{F} 0$ rabbits at Tyr-1 p. Q68stop and Lmna-1 p. G607G loci. These results indicate that these efficient and precise base editors can be used as a reliable tool for inducing highprecision base editing in rabbits. Furthermore, a similar strategy can be widely used in other organisms in the future.

In addition to accuracy, the genome-targeting scope also represents a primary obstacle for base editors. To date, a variety of SpCas9 homologs and variants that recognize a variety of PAMs were found, such as SaCas9 $(\mathrm{NNGRRT})^{19}, \mathrm{Cpf1}(\mathrm{TTTV})^{20}$, ScCas9 $(\mathrm{NNG})^{21}$, Nme2Cas9 (N4CC) ${ }^{22}$, SpCas9-VQR (NGA) ${ }^{23}$, and SpCas9-NG $(\mathrm{NGN})^{24}$. It may further improve the accuracy and genome-targeting scope of base editing to combine these variants with the YFE system.

Moreover, a novel BE architecture with an engineered human APOBEC3A (eA3A) domain offered an alternative strategy to further reduce bystander mutations, but the application of this system was restricted by the presence of TCR (A/G) motifs ${ }^{5}$. Context-dependent base editors represent an important advance that offers more precise base editing, whereas may lower the target site applicability because the target nucleotide must naturally exist in the preferred sequence context ${ }^{25}$. Therefore, selecting 


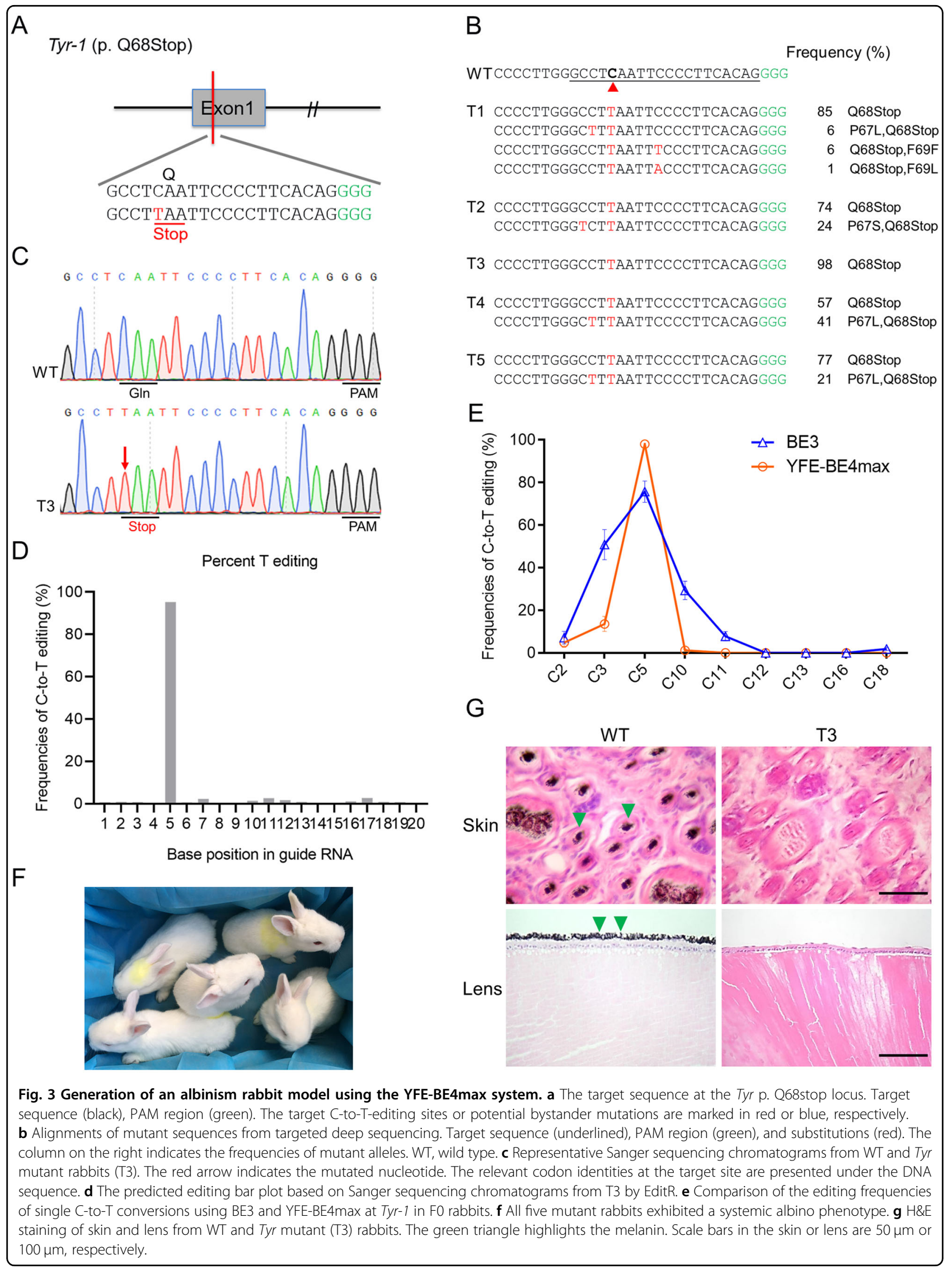


A Lmna-1 (p. G607G)

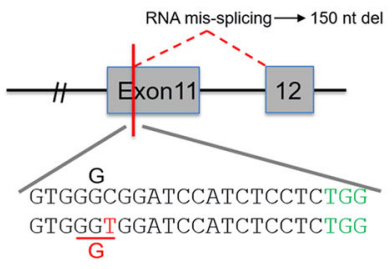

C
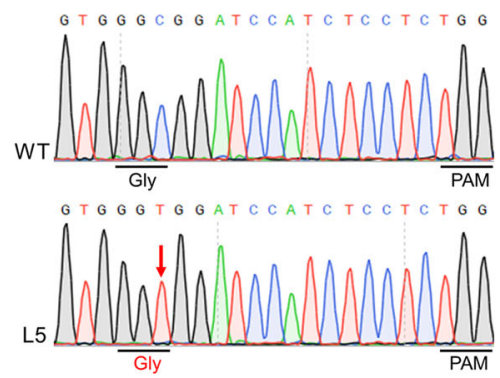

D

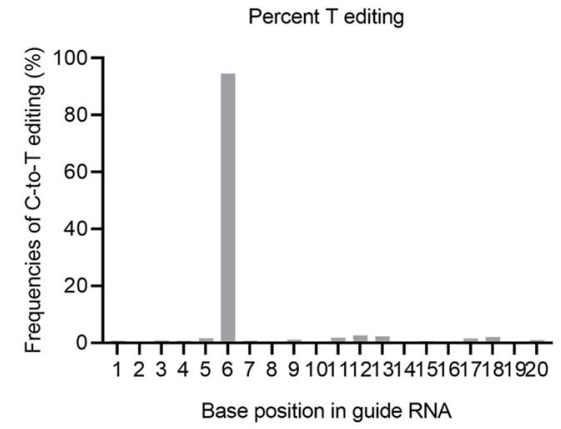

G

DL 2000 L1 L2 L3 $\quad$ L4 $\quad$ L5 $\quad$ L6 $\quad$ WT1WT2

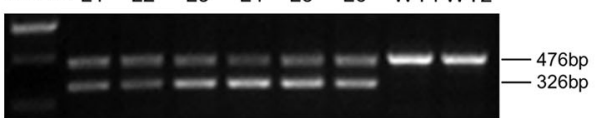

$\mathrm{H}$

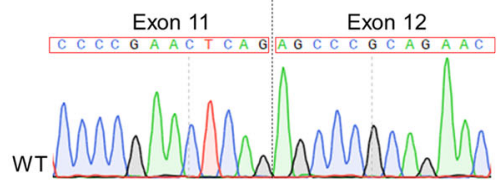

$$
\begin{array}{c:r}
\text { Exon } 11-150 \mathrm{bp} & \text { Exon } 12 \\
\hline C C G G G A G C C C A G A C C C G C A G
\end{array}
$$

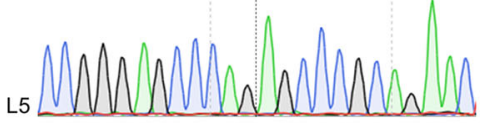

B

WTGAGCCCAGGTGGGCGGATCCATCTCCTCTGG

Frequency (\%)

L1 GAGCCCAGGTGGGTGGATCCATCTCCTCTGG

57 G607G

L2 GAGCCCAGGTGGGTGGATCCATCTCCTCTGG

63 G607G

L3 GAGCCCAGGTGGGTGGATCCATCTCCTCTGC

91 G607G

L4 GAGCCCAGGTGGGTGGATCCATCTCCTCTGG

79 G607G

L5 GAGCCCAGGTGGGTGGATCCATCTCCTCTGG

98 G607G

L6 GAGCCCAGGTGGGTGGATCCATCTCCTCTGG GAGCCCAGGTGGGTGGATTCATCTCCTCTGC

91 G607G

7 G607G,S609F

E

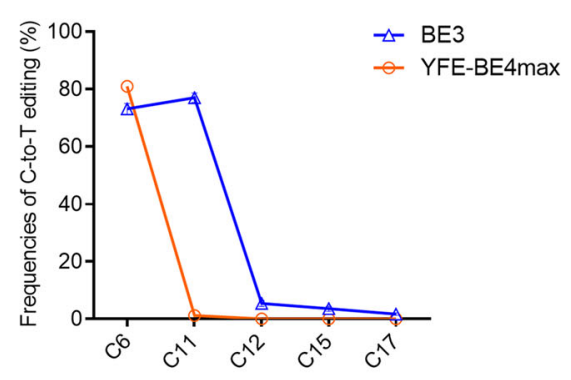

F

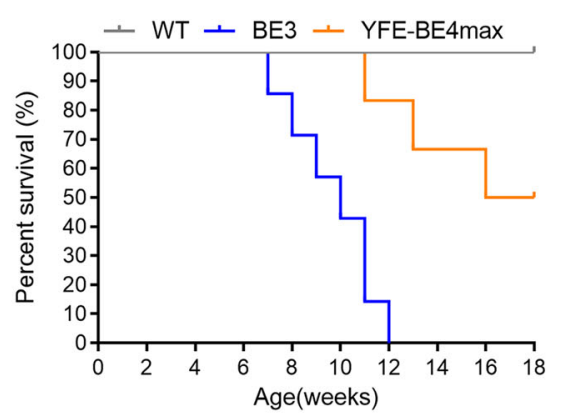

I

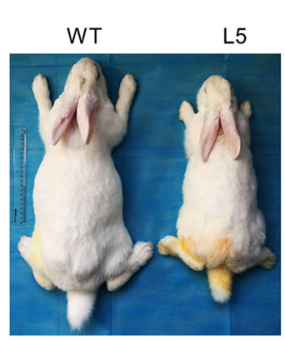

J

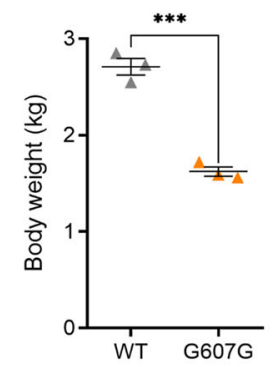

K
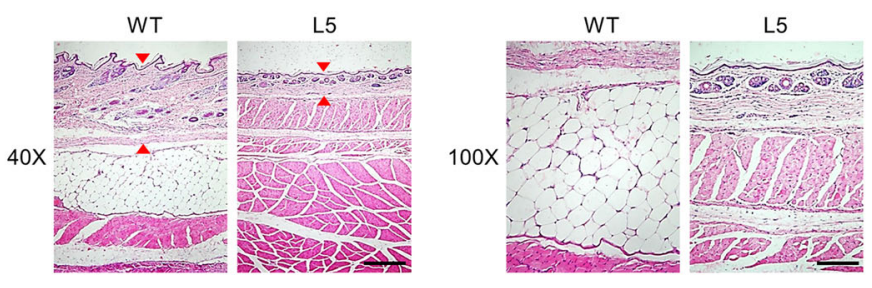

Fig. 4 (See legend on next page.) 
(see figure on previous page)

Fig. 4 Generation of an HGPS rabbit model using the YFE-BE4max system. a The target sequence at the $L m n a$ p. G607G locus. Target sequence (black), PAM region (green). The targeted C-to-T-editing site and potential bystander mutations are marked in red or blue, respectively. b Alignments of mutant sequences from targeted deep sequencing. Target sequence (underlined), PAM region (green), and substitutions (red). The column on the right indicates the frequencies of mutant alleles. WT, wild type. c Representative Sanger sequencing chromatograms from WT and Lmna mutant rabbits (L5). The red arrow indicates the mutated nucleotide. The relevant codon identities at the target site are presented under the DNA sequence. d The predicted editing bar plot based on Sanger sequencing chromatograms from $L 5$ by EditR. e Comparison of the editing frequencies of single Cto-T conversions using BE3 and YFE-BE4max at Lmna- 1 in F0 rabbits. $\mathbf{f}$ Survival curves of WT and F0 mutant rabbits produced by the BE3 and YFEBE4max systems. g Demonstration of the abnormal splicing product using RT-PCR, showing a spliced product of 326 bp in mutants (L1-L6) rather than WT rabbits. $\mathbf{h}$ Sanger sequencing chromatograms of the abnormal RT-PCR product confirmed the deletion of 150 nucleotides within exon 11 in the mutant rabbit (L5). i Photograph of WT and $L m n$ a mutant (L5) rabbits at 3 months. $\mathbf{j}$ Quantification and comparison of the body weight between WT and Lmna mutant rabbits at 3 months. ${ }^{* *} p<0.001$, Student's $t$ test. $\mathbf{k}$ H\&E staining of the skin from a 3-month-old Lmna mutant (L5) compared with that of a WT rabbit. The red triangle highlights the thin skin. Scale bars at $\times 40$ and $\times 100$ are $500 \mu \mathrm{m}$ and $200 \mu \mathrm{m}$, respectively.

reasonable base editors with narrowed window or context-specificity to preferentially edit the target base over the bystander base may be the future approach.

In summary, our optimized YFE system is a precise base editing tool that has significantly reduced bystander activity and high editing efficiency. In addition, YFE system could be used to precisely mimic human pathogenic mutations by inducing $\mathrm{C}$-to- $\mathrm{T}$ base conversions in rabbits. Overall, these engineered Cas9-cytidine deaminase fusions are promising tools for animal model establishment and precise gene therapy in the future.

\section{Materials and methods}

\section{Ethics statement}

New Zealand white and Lianshan black rabbits were obtained from the Laboratory Animal Center of Jilin University (Changchun, China). All animal studies were conducted according to experimental practices and standards approved by the Animal Welfare and Research Ethics Committee at Jilin University (IACUC number: KT201801001).

\section{Plasmid construction}

The BE4-Gam and BE4max plasmids were obtained from Addgene (\#100806 and \#112093). The corresponding mutations were introduced into BE4-Gam or BE4max to obtain YE1 base editors (W90Y + R126E in rA1), YEE base editors (W90Y + R126E + R132E in rA1), Y120Fbase editors (Y120F in rA1) and YFE-base editors (W90Y + Y120F + R126E in rA1). Plasmid site-directed mutagenesis was performed using the Fast Site-Directed Mutagenesis Kit (TIANGEN, Beijing). All site-directed mutation primers are listed in Table S1.

\section{mRNA and gRNA preparation}

All plasmids were linearized with NotI and transcribed in vitro using the HiScribe T7 ARCA mRNA Kit (NEB). The RNeasy Mini Kit (Qiagen) was used for mRNA purification according to the manufacturer's instructions. The sgRNA oligos were annealed into pUC57-sgRNA expression vectors containing a T7 promoter. The
sgRNAs were then amplified and transcribed in vitro using the MAXIscript T7 Kit (Ambion) and purified using the miRNeasy Mini Kit (Qiagen) according to the manufacturer's instructions. The sgRNA oligo sequences used in this study are listed in Table S2.

\section{Microinjection of rabbit zygotes and embryo transfer}

The protocol used for the microinjection of pronuclearstage embryos has been described in detail in our previously published study ${ }^{26}$. In brief, a mixture of mRNA $(200 \mathrm{ng} / \mu \mathrm{l})$ and sgRNA $(50 \mathrm{ng} / \mu \mathrm{l})$ was co-injected into the cytoplasm of pronuclear-stage zygotes. The injected embryos were transferred into Earle's Balanced Salt Solution (EBSS) medium for short-term culture at $38.5^{\circ} \mathrm{C}$, $5 \%$ carbon dioxide and $100 \%$ humidity. Then, $\sim 30-50$ injected zygotes were transferred into the oviducts of recipient rabbits.

\section{Single-embryo PCR amplification and rabbit genotyping}

Each group of base editing was injected with BEencoding mRNA and corresponding sgRNA using an average of 10 embryos to test the base editing efficiency. The injected embryos were transferred into EBSS medium for culture at $38.5^{\circ} \mathrm{C}, 5 \%$ carbon dioxide, and $100 \%$ humidity. Then, the injected embryos were collected at the blastocyst stage. Genomic DNA was extracted in embryo lysis buffer $(1 \% \mathrm{NP} 40)$ at $56^{\circ} \mathrm{C}$ for $60 \mathrm{~min}$ and then at $95^{\circ} \mathrm{C}$ for $10 \mathrm{~min}$ in a BIO-RAD PCR Amplifier. Then the extracted products were amplified by $\mathrm{PCR}$ $\left(95^{\circ} \mathrm{C}, 5 \mathrm{~min}, 42\right.$ cycles of $\left(95^{\circ} \mathrm{C}, 30 \mathrm{~s}, 58^{\circ} \mathrm{C}, 30 \mathrm{~s}, 72^{\circ} \mathrm{C}\right.$, $30 \mathrm{~s}), 72^{\circ} \mathrm{C}, 5 \mathrm{~min}$ ) and determined by Sanger sequencing. The Sanger sequencing result of each blastocyst was used to evaluate base editing frequencies or indel frequencies by $\operatorname{EditR}^{8}$. The genomic DNA of newborn rabbits was extracted from ear clips and analyzed by PCR genotyping, Sanger sequencing and targeted deep sequencing. All primers used for genotyping are listed in Table S3.

\section{Targeted deep sequencing}

Targeted sites were amplified from genomic DNA using Phusion polymerase (Thermo Fisher Scientific). The 
paired-end sequencing of PCR amplicons was performed by Sangon Biotech (Shanghai) using an Illumina MiSeq. The average depth of coverage for the sequencing runs was $\sim 20,000$ reads.

\section{Off-target assay}

The top ten potential off-target sites in the rabbit genome for sgRNA were predicted to analyze site-specific edits according to Cas-OFFinder ${ }^{27}$ (http://www.rgenome. net/cas-offinder/). All primers for the off-target assay are listed in Tables S4 and S5.

\section{H\&E staining}

The dorsal skin and lens from WT and mutant rabbits were fixed in $4 \%$ paraformaldehyde for $48 \mathrm{~h}$, embedded in paraffin wax and subsequently sectioned for slides. The slides were stained with $H \& E$ and viewed under a Nikon TS100 inverted microscope.

\section{Statistical analysis}

All data are expressed as the mean \pm SEM of at least three individual determinations for all experiments. Data were analyzed by Student's $t$ test via GraphPad prism software 6.0. Probability values $<0.05(p<0.05)$ were considered to be statistically significant. ${ }^{*} p<0.05$, ${ }^{* * *} p<$ $0.01,{ }^{* * * *} p<0.001,{ }^{* * * * * *} p<0.0001$.

\section{Acknowledgements}

We thank Peiran Hu and Nannan Li for assistance at the Embryo Engineering Center for the critical technical assistance. This study was financially supported by the National Key Research and Development Program of China Stem Cell and Translational Research (2017YFA0105101). The Program for Changjiang Scholars and Innovative Research Team in University (No.IRT_16R32). The Strategic Priority Research Program of the Chinese Academy of Sciences (XDA16030501, XDA16030503), Key Research \& Development Program of Guangzhou Regenerative Medicine and Health Guangdong Laboratory (2018GZR110104004).

\section{Author details \\ ${ }^{1}$ Key Laboratory of Zoonosis Research, Ministry of Education, College of Animal Science, Jilin University, Changchun 130062, China. ${ }^{2}$ CAS Key Laboratory of Regenerative Biology, Guangdong Provincial Key Laboratory of Stem Cell and Regenerative Medicine, South China Institute for Stem Cell Biology and Regenerative Medicine, Guangzhou Institutes of Biomedicine and Health, Chinese Academy of Sciences, Guangzhou 510530, China. ${ }^{3}$ Guangzhou Regenerative Medicine and Health Guang Dong Laboratory (GRMH-GDL), Guangzhou 510005, China. ${ }^{4}$ Institute for Stem Cell and Regeneration, Chinese Academy of Sciences, Beijing 100101, China}

\section{Author contributions}

Z. Liu, L. Lai, and Z. Li conceived and designed the experiments. Z. Liu, S. Chen, and H. Shan performed the experiments. Z. Liu, S. Chen, H. Shan analyzed the data. Y. Jia, M. Chen, and Y. Song contributed reagents/materials/analysis tools. Z. Liu and Z. Li wrote the paper. All authors have read and approved the final manuscript.

\section{Data availability}

The authors state that all data necessary for confirmation of the conclusions presented in the article are represented fully within the article or are available from the authors upon request.
Conflict of interest

The authors declare no conflict of interest.

\section{Publisher's note}

Springer Nature remains neutral with regard to jurisdictional claims in published maps and institutional affiliations.

Supplementary Information accompanies this paper at (https://doi.org/ 10.1038/s41419-020-2244-3).

Received: 31 October 2019 Revised: 7 January 2020 Accepted: 9 January 2020

Published online: 20 January 2020

\section{References}

1. Cong, L. et al. Multiplex genome engineering using CRISPR/Cas systems. Science 339, 819-823 (2013).

2. Knott, G. J. \& Doudna, J. A. CRISPR-Cas guides the future of genetic engineering. Science 361, 866-869 (2018).

3. Komor, A. C., Kim, Y. B., Packer, M. S., Zuris, J. A. \& Liu, D. R. Programmable editing of a target base in genomic DNA without double-stranded DNA cleavage. Nature 533, 420-424 (2016).

4. Kim, Y. B., Komor, A. C. \& Levy, J. M. Increasing the genome-targeting scope and precision of base editing with engineered Cas9-cytidine deaminase fusions. Nat. Biotechnol. 35, 371-376 (2017).

5. Gehrke, J. M., Cervantes, O. \& Clement, M. K. An APOBEC3A-Cas9 base editor with minimized bystander and off-target activities. Nat. Biotechnol. 36, 977-982 (2018).

6. Liu, Z. et al. Highly efficient RNA-guided base editing in rabbit. Nat. Commun. 9, 2717 (2018).

7. Komor, A. C., Zhao, K. T. \& Packer, M. S. Improved base excision repair inhibition and bacteriophage Mu Gam protein yields C:G-to-T:A base editors with higher efficiency and product purity. Sci. Adv. 3, eaao4774 (2017).

8. G, K. M. et al. EditR: a method to quantify base editing from sanger sequencing. CRISPR J. 1, 239-250 (2018).

9. Coelho, M. A. et al. BE-FLARE: a fluorescent reporter of base editing activity reveals editing characteristics of APOBEC3A and APOBEC3B. BMC Biol. 16, 150 (2018).

10. Li, X., Wang, Y., Liu, Y. \& Yang, B. Base editing with a Cpf1-cytidine deaminase fusion. Nat. Biotechnol. 36, 324-327 (2018).

11. Koblan, L. W., Doman, J. L. \& Wilson, C. Improving cytidine and adenine base editors by expression optimization and ancestral reconstruction. Nat. Biotechnol. 36, 843-846 (2018).

12. Wang, X., Li, J., Wang, Y. \& Yang, B. Efficient base editing in methylated regions with a human APOBEC3A-Cas9 fusion. Nat. Biotechnol. 36, 946-949 (2018).

13. Shi, K. et al. Structural basis for targeted DNA cytosine deamination and mutagenesis by APOBEC3A and APOBEC3B. Nat. Struct. Mol. Biol. 24, 131-139 (2017).

14. Kouno, T. et al. Crystal structure of APOBEC3A bound to single-stranded DNA reveals structural basis for cytidine deamination and specificity. Nat. Commun. 8, 15024 (2017).

15. Oetting, W. S. The tyrosinase gene and oculocutaneous albinism type 1 (OCA1): a model for understanding the molecular biology of melanin formation. Pigment Cell Res. 13, 320-325 (2000).

16. Pollex, R. L. \& Hegele, R. A. Hutchinson-Gilford progeria syndrome. Clin. Genet. 66, 375-381 (2004).

17. Gonzalo, S., Kreienkamp, R. \& Askjaer, P. Hutchinson-Gilford Progeria Syndrome: a premature aging disease caused by LMNA gene mutations. Ageing Res. Rev. 33, 18-29 (2017).

18. Schreiber, K. H. \& Kennedy, B. K. When lamins go bad: nuclear structure and disease. Cell 152, 1365-1375 (2013).

19. Ran, F. A. et al. In vivo genome editing using Staphylococcus aureus Cas9. Nature 520, 186-191 (2015).

20. Zetsche, B. et al. Cpf1 is a single RNA-guided endonuclease of a class 2 CRISPR-Cas system. Cell 163, 759-771 (2015).

21. Chatterjee, P., Jakimo, N. \& Jacobson, J. M. Minimal PAM specificity of a highly similar SpCas9 ortholog. Sci. Adv. 4, eaau0766 (2018).

22. Edraki, A. et al. A compact, high-accuracy Cas9 with a dinucleotide PAM for in vivo genome editing. Mol. Cell 73, 714-726.e714 (2019). 
23. Kleinstiver, B. P. et al. Engineered CRISPR-Cas9 nucleases with altered PAM specificities. Nature 523, 481-485 (2015).

24. Nishimasu, H. \& Shi, X. Engineered CRISPR-Cas9 nuclease with expanded targeting space. Science 361, 1259-1262 (2018).

25. Rees, H. A. \& Liu, D. R. Base editing: precision chemistry on the genome and transcriptome of living cells. Nat. Rev. Genet. 19, 770-788 (2018).
26. Song, Y. et al. Efficient dual sgRNA-directed large gene deletion in rabbit with CRISPR/Cas9 system. Cell. Mol. Life Sci. 73, 2959-2968 (2016).

27. Bae, S., Park, J. \& Kim, J. S. Cas-OFFinder: a fast and versatile algorithm that searches for potential off-target sites of Cas9 RNA-guided endonucleases. Bioinformatics 30, 1473-1475 (2014). 